Check for updates

Cite this: RSC Adv., 2019, 9, 23554

\title{
Self-powered gas sensor based on SiNWs/ITO photodiode $\uparrow$
}

\author{
Dong Liu, ${ }^{a}$ Qiaofen Chen, ${ }^{a}$ Aimin Chen ${ }^{b}$ and Jianmin Wu (D) *a
}

Silicon nanowires (SiNWs) with a large surface-to-volume ratio and a low reflectivity are exceedingly attractive building blocks for developing high performance light harvesting devices. Herein, a SiNW/ITO heterojunction was fabricated easily by just compressing the SiNWs and ITO electrode together with a suitable pressure. Under light illumination, the SiNWs/ITO with an optimized structure can generate more than $20 \mu \mathrm{A}$ photocurrent at zero bias voltage. In the mean time, the photocurrent is very sensitive to $\mathrm{NO}_{2}$ infiltration into the forest of SiNWs and displays a non-linear relationship with the concentration of $\mathrm{NO}_{2}$ from 0 to $1000 \mathrm{ppb}$. In comparison with chemiresistive sensors based on SiNWs only, the sensitivity of the self-powered sensor was improved obviously, showing a limit of detection at $\sim 5 \mathrm{ppb}$. The excellent light trapping and sensing performance was attributed to the heterojunction formed between SiNWs and ITO. Since the nano-photodiode device can monitor the surrounding gas without an external power supply, it will ensure that sensor networks can operate independently and sustainably without a battery or at least by extending the life time of a battery. This work may push forward the development of self-powered microsensors using rationally designed nanojunctions.

Received 21st April 2019

Accepted 23rd July 2019

DOI: 10.1039/c9ra02972a

rsc.li/rsc-advances
Up to now, the self-powered nanosystem known as an active sensor, has been achieved by using piezoelectric nanogenerators as either a power source or sensing signal in response to the change in environment, such as self-powered wind-velocity detectors, automobile speedometers, gas sensors, and magnetic sensors. ${ }^{\mathbf{1 0 - 1 3}}$ The solar driven self-power sensor is another kind of promising strategy, which uses a multifunctional heterostructure to harvest solar light for driving the sensor in one hand and providing good sensing properties on another hand. ${ }^{14}$

Silicon nanowire (SiNW) arrays with their unique onedimensional aligned structure and outstanding electrical properties, ${ }^{15}$ have attracted much attention because they are important photoelectric conversion materials with high optical absorption ability. For example, large-area ordered SiNW arrays prepared by electroless etching could intensively suppress light reflection over a wide spectral range from 300 to $1000 \mathrm{~nm} .{ }^{16}$ The remarkable antireflection ability of the SiNW arrays has a great advantage over other texturized Si and other types of antireflection coating materials. ${ }^{17}$ More importantly, various types of heterojunctions and homojunctions can be constructed from SiNWs with different doping types and doping levels. ${ }^{18} \mathrm{~A}$ few recent studies have also implemented self-powered devices which are activated by light based on a $\mathrm{p}-\mathrm{n}$ junction. ${ }^{19,20}$ The existing built-in electric field (BEF) can separate photogenerated carriers which facilitates the driving of devices under light illumination. For example, Hoffmann et al. ${ }^{21,22}$ reported two kinds of self-powered gas sensors using n-ZnO/p-Si and CdS@n$\mathrm{ZnO} / \mathrm{p}-\mathrm{Si}$, respectively. Yi Jia et al. utilize SiNW arrays to
${ }^{a}$ Institute of Analytical System, Department of Chemistry, Zhejiang University, Hangzhou, 310058, China. E-mail: wjm-st1@zju.edu.cn

${ }^{b}$ College of Chemical Engineering, Zhejiang University of Technology, Hangzhou 310014, China

† Electronic supplementary information (ESI) available. See DOI: 10.1039/c9ra02972a 
fabricate heterojunction with CNT membranes, which combines the functions of solar cell and gas sensor. ${ }^{23}$

In the present work, p-SiNWs and n-ITO were employed to fabricate the self-powered gas sensor with a simple $\mathrm{p}-\mathrm{n}$ heterojunction structure which was activated by a light source. In this structure, the large specific surface area of SiNW arrays can greatly facilitate gas adsorption and desorption, further improving the sensing performances. Compared with the conventional chemiresistive gas sensor using SiNWs, the sensor reported here was capable of detecting $\mathrm{NO}_{2}$ concentrations at the sub-ppm level with a very high selectivity at room temperature (RT) and show a linear response to $50-1000 \mathrm{ppb} \mathrm{NO}_{2}$ when illuminated under visible light $\left(\lambda=576 \mathrm{~nm}, P=20 \mathrm{~mW} \mathrm{~cm}^{-2}\right)$. More importantly, the sensor can generate responding signal without need of an external power-source. The current results could stimulate a feasible approach for the next generation of portable low-temperature self-powered gas sensors.

\section{Results and discussion}

The schematic experimental setup is illustrated in Fig. 1. The SiNWs/ITO structure is basically constructed by compressing the transparent ITO and the tip of SiNWs with proper pressure. Such a structure allows sunlight to pass towards the SiNWs array. In order to investigate the performance of gas sensing, the SiNWs/ITO sensor was sealed into a chamber with a window for light illumination. The concentration of $\mathrm{NO}_{2}$ gas sample was adjusted by mixing the standard $\mathrm{NO}_{2}$ sample (16.8 ppm) with $\mathrm{N}_{2}$ at a specific ratio controlled by an automatic gas mixing apparatus. The sealed chamber was kept at room temperature. The current-voltage $(I-V)$ data of SiNW/ITO heterojunction were recorded by a Keithley 6487 digital source-meter.

The top view of SiNWs with low magnification shows that the as prepared SiNWs display high density and highly ordered structure over a large area (Fig. 2a). The cross-sectional view (Fig. 2b) indicates that semi-densely distributed SiNWs are oriented and vertical to the Si wafer substrate. The average length of the SiNWs prepared by $2 \mathrm{~h}$ electroless etching is about $37.5 \mu \mathrm{m}$ and the diameters of SiNWs range from 50 to $200 \mathrm{~nm}$. Minor broken SiNWs were caused by cutting and loading of the SEM specimens. With the increase of the etching time, the length of SiNWs increased linearly (Fig. S1†).

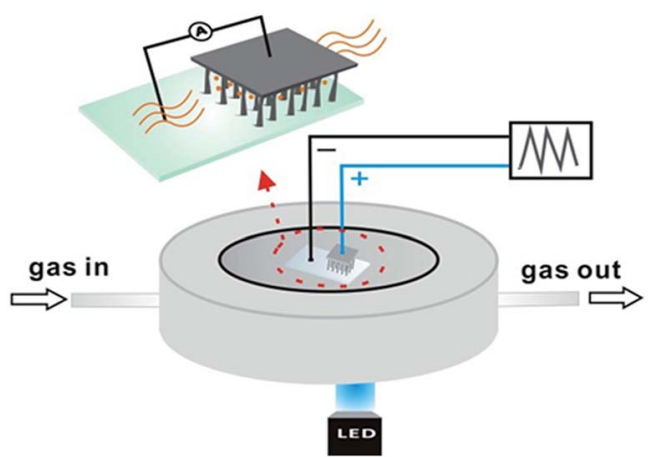

Fig. 1 Schematic diagram of the p-SiNW/ITO structure, the inset shows details of the heterojunction fabrication.

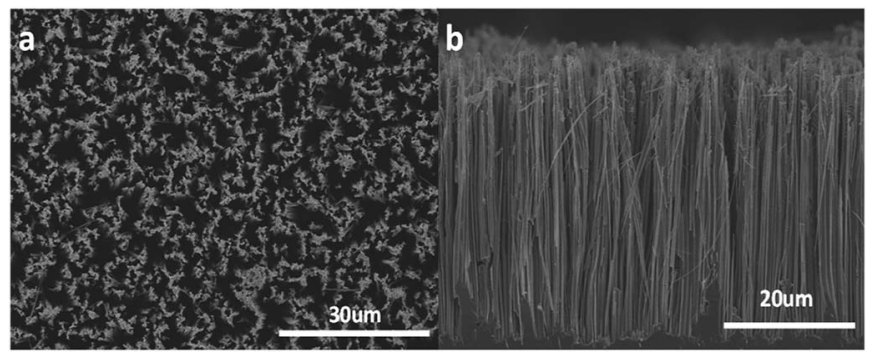

Fig. 2 (a) Top view and (b) cross-sectional view SEM images of a SiNW arrays, the etching time was $2 \mathrm{~h}$.

Current-voltage $(I-V)$ characteristics of the n-SiNWs/ITO device shows a symmetrical current response under both forward and reverse voltage scanning, even though a non-linear current response was observed owing to the formation of homojunction. Under light illumination, the n-SiNWs/ITO still displayed a symmetrical $I-V$ curve (Fig. 3a). As shown in the zoomed in area ranging from $-0.6 \mathrm{~V}$ to $0.6 \mathrm{~V}$ (Fig. $3 \mathrm{~b}$ ), the $\mathrm{n}$ SiNWs/ITO cannot generate photocurrent at zero bias voltage, indicating that these kinds of structure cannot act as a selfpowered device. In contrast, the $I-V$ characteristics of $\mathrm{p}$ SiNWs/ITO in dark and under illumination in nitrogen atmosphere show a significant rectifying effect (Fig. 3c), which was originated from the heterojunction at the p-SiNWs/ITO interface. In dark conditions, p-SiNWs/ITO showed typical rectifying diode behavior, while light illumination shifts the device characteristics to a photovoltaic effect. On the p-SiNWs/ITO heterojunctions, the current observed at light irradiation was significantly larger than that under dark condition when a negative voltage was applied, indicating the generation of photoexcited charge carriers at the heterojunctions. Even at zero voltage, a large photocurrent $(>20 \mu \mathrm{A})$ was observed
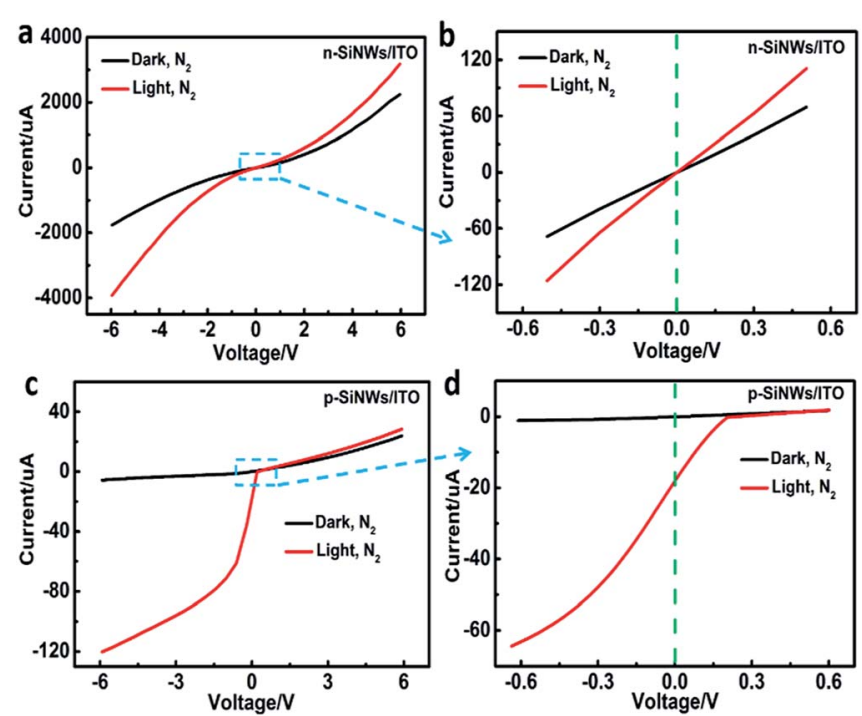

Fig. 3 The current-voltage $(I-V)$ curve of SiNWs/ITO heterojunction (a) n-type and (c) p-type SiNWs under dark (black) and visual LED (red) conditions and (b), (d) the amplification of (a), (c) respectively under $\mathrm{N}_{2}$ atmosphere, the etching time of SiNWs was $2 \mathrm{~h}$. 
(Fig. 3d). Under positive voltage, the current response at light irradiation was only slightly larger than that at dark condition, indicating that negative voltages applied to the heterojunctions favour the generation of photocurrent. The phenomenon is in accordance with conventional semiconductor photodiode, which display a directional photocurrent response. Therefore, the p-SiNWs/ITO device was chosen as the self-powered device for gas sensing. Meanwhile, the photocurrent produced by this structure can sustain for a long time (Fig. S2 $\dagger$ ).

The device exhibited different photoresponse when the SiNWs length varied (Fig. 4a-d). The length of SiNWs was controlled by the etching time since the length of SiNWs have a proportional relationship with the etching time. In the present work, the etching time was set at $30 \mathrm{~min}, 1 \mathrm{~h}, 1.5 \mathrm{~h}, 2 \mathrm{~h}$ and $3 \mathrm{~h}$, respectively. SEM indicated that the length of SiNWs increased with the increasing etching time (Fig. S1 $\dagger$ ). The $I-V$ curves of SiNWs/ITO heterojunction with different SiNWs length in the dark and illumination indicated that the photoresponse increased with the increasing etching time from 0 to $2 \mathrm{~h}$ (Fig. $4 \mathrm{a}$ and $\mathrm{b}$ ), but decreased when the etching time exceeded $2 \mathrm{~h}$. The photocurrent of SiNWs/ITO with different length of SiNWs under zero bias voltage showed repeated response when illumination was cyclically turned on $(100 \mathrm{~s})$ and off $(100 \mathrm{~s})$ by three times (Fig. $4 \mathrm{c})$. The orders of photosensitivity $\left(I_{\text {light }} / I_{\text {dark }}\right)$ is in accordance with the results obtain in $I-t$ curve measurements (Fig. 4d). Compared with silicon wafer, the $I_{\text {light }} / I_{\text {dark }}$ value of SiNWs increased two orders because the light reflectance of SiNW arrays is significantly lower (Fig. S3†), thereby displaying superior light trapping effect. The reflectance of SiNWs array further decreased with increase of SiNWs length owing to multiple reflections in the forest of SiNW arrays. ${ }^{24}$ However, the $I_{\text {light }} / I_{\text {dark }}$ reaches a maximal value after $2 \mathrm{~h}$ etching time, thereafter, the photoresponse of the heterojunction decreases significantly. This phenomenon might be caused by the
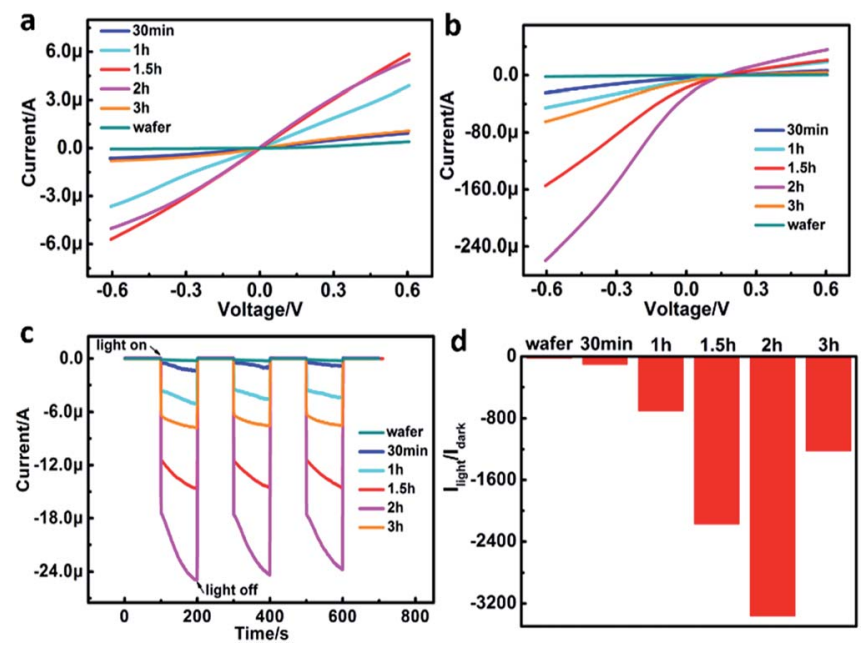

Fig. 4 The $I-V$ curve of $\mathrm{p}$-SiNWs/ITO heterojunction under (a) dark and (b) visible LED conditions, (c) photocurrent under zero bias when illumination is turned on (100 s) and off (100 s), repeated for three cycles, (d) photosensitivity of heterojunctions with different length of SiNWs prepared by different etching time (wafer, $30 \mathrm{~min}, 1 \mathrm{~h}, 1.5 \mathrm{~h}, 2 \mathrm{~h}$, 3 h). competition between improved light absorption and increased electron-hole recombination in SiNWs when the length of SiNWs increase. ${ }^{25}$ In other words, longer SiNWs are able to absorb more photons and thus generate more charge carriers. However, charge traps and scattering centers are simultaneously increase in longer SiNWs, leading to more recombination. As a result, a length for maximum photoresponse needs to be optimized. In this work, the optimum length of SiNWs for maximum photoresponse was $\sim 37 \mu \mathrm{m}$.

To determine the sensing characteristics of the self-powered sensor, the $I-V$ characteristics of p-SiNWs/ITO heterojunction under $\mathrm{N}_{2}$ and 5 ppm $\mathrm{NO}_{2}$ atmosphere were measured respectively (Fig. $5 \mathrm{a}$ and $\mathrm{b}$ ). The $I-V$ curves showed different behavior when the device was alternatively exposed to $\mathrm{N}_{2}$ and $\mathrm{NO}_{2}$ under dark (black and blue) and illumination (red and purplish red) conditions. As shown in Fig. 5a, upon exposure to 5 ppm $\mathrm{NO}_{2}$, the photocurrent sharply drops at negative voltage than that positive voltage under illumination. However, under the dark condition, the photocurrent response at negative voltage is almost the same as that at positive voltage (Fig. 5b). The result indicated that the photocurrent generated by the heterojunction between nanowire and ITO can sensitively respond to the gas dosing. The open-circuit current-time $(I-t)$ curve of the device under light illumination $\left(\lambda=576 \mathrm{~nm}, P=20 \mathrm{~mW} \mathrm{~cm}^{-2}\right)$ was obtained at zero bias voltage (Fig. $5 \mathrm{c}$ ). Before the dosing of $\mathrm{NO}_{2}$ gas, the device was equilibriumed in dry $\mathrm{N}_{2}$ gas under dark condition. Upon turning on light, the device produced an $8 \mu \mathrm{A}$ negative photocurrent. After exposing with $\mathrm{NO}_{2}$, the sensor displayed positive photocurrent response, which increased with the increasing concentration of $\mathrm{NO}_{2}$ gas. The response $(R)$ of the self-power $\mathrm{NO}_{2}$ sensor was defined according to the following equation:

$$
R=\left(I-I_{0}\right) / I_{0}
$$
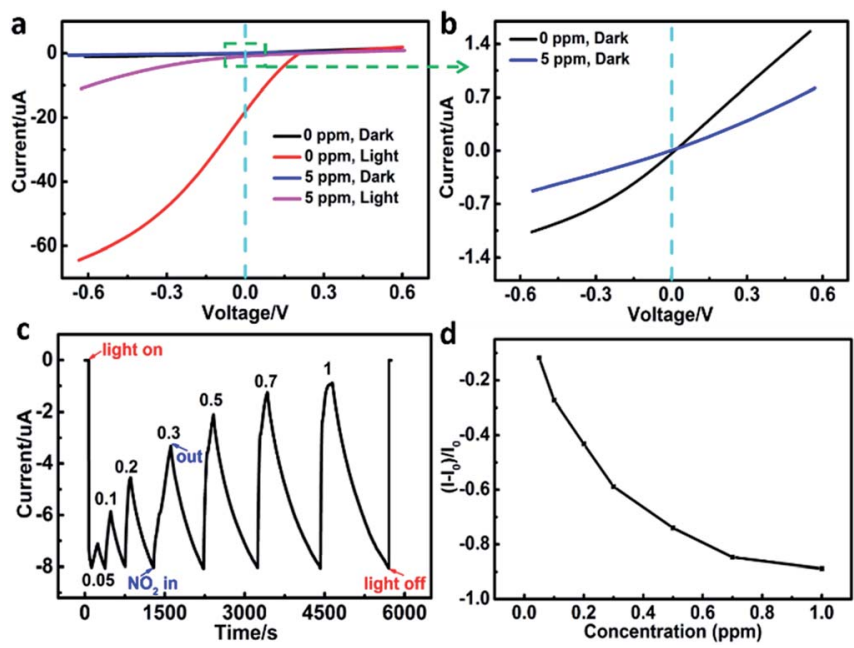

Fig. 5 (a) Dark and light $I-V$ curves of $p$-SiNWs/ITO heterojunction before (black, red) and after (blue, purplish red) 5 ppm NO $\mathrm{N}_{2}$ importing respectively, (b) the amplification of the $I-V$ curve in dark condition (c) real-time $\mathrm{NO}_{2}$ detection with different concentrations (ppm) of $\mathrm{p}$ SiNWs/ITO heterojunction (d) the linear relationship between the relative response and concentration, the SINWs etching time was $2 \mathrm{~h}$. 
where $I_{0}$ and $I$ are the currents before and after $\mathrm{NO}_{2}$ exposure at zero bias under illumination at room temperature. The $R v s$. $c$ $\left(\mathrm{NO}_{2}\right)$ is plotted in Fig. $5 \mathrm{~d}$, which shows an exponential relationship within the concentration from 0.05-1 ppm. According to the calibration curve and signal noise measured in photocurrent baseline, the detection limit of the self-powered sensor is as low as $5 \mathrm{ppb}$ for $\mathrm{NO}_{2}$. Response and recovery time $\left(t_{\mathrm{res}}\right.$ and $\left.t_{\text {rec }}\right)$ are defined as the time it takes for the resistance to reach $90 \%$ of its steady-state value after introduction or removal of the analyte gas, respectively. As shown in Fig. $\mathrm{S} 4, \dagger t_{\text {res }}$ and $t_{\text {rec }}$ are $\sim 80 \mathrm{~s}$ and $850 \mathrm{~s}$ for the $\mathrm{p}$-SiNWs/ITO sensor to $1 \mathrm{ppm} \mathrm{NO}_{2}$. In addition, the self-powered sensor chips showed good reproducibility no matter the same chip or different chips (Fig. S5 and $56 \dagger$ ). In contrast, the gas sensing performance of pure $\mathrm{p}$ type SiNWs sensor in response to $\mathrm{NO}_{2}$ was much lower (Fig. S7†).

\section{Sensing mechanism}

The high photosensitivity and gas sensitivity of the device are attributed to the photocatalysis of HF-treated SiNWs and the combined actions of the SiNWs and ITO. For the photosensitivity performance, except the excellent light reflection suppression effect of SiNWs, the HF-treated process was also very important. If the SiNWs weren't treated by the HF, the photocurrent can't be sustainable (Fig. S8 $\dagger$ ). This is because hydrogen atom has a large Pauling electron negative value of 2.2; consequently, the terminated hydrogen atom with a charge of 0.09-0.13 au may serve as an electron sink and accelerate the separation of the photoinduced electron and hole thus promote the photocatalytic efficiency. ${ }^{26}$ Beside above factors, the built-in electric field within the p-SiNWs array/ITO junction could also guarantee both a high photocurrent and a low dark current (10 nA at zero bias, Fig. 5c). For a pure p-type SiNWs gas sensors, the resistance would decrease when importing the oxidizing gases due to the increase of majority carriers. However, in this selfpowered device, the photocurrent decreased in response to $\mathrm{NO}_{2}$, inferring that the mechanism of this structure cannot be explained just by the carrier change of SiNWs owing to the formation of $\mathrm{p}-\mathrm{n}$ heterojunctions between $\mathrm{p}$-SiNWs and ITO. Because of the different work function between p-SiNWs $(\sim 1.12$ $\mathrm{eV})^{27}$ and ITO $(\sim 3.5 \mathrm{eV}){ }^{28}$ the carriers diffusion under thermal equilibrium conditions lead to the formation of a built-in electric field with a direction from ITO to p-SiNWs. The energy band diagram and its photovoltaic characteristics of the self-powered p-SiNWs/n-ITO are illustrated in Fig. 6. In the dark condition, the small forward bias could produce a positive current in the circuit. However, upon light illumination, the photoinduced electromotive force was in the reverse direction to the built-in bias, and therefore led to the current change from positive to negative. When illuminated by light, electron-hole pairs generate in the SiNWs and then inject the excited electrons $\left(\mathrm{e}^{-}\right)$from the conduction band of SiNWs into the conduction band of ITO, whilst the mismatch in the valence band and absence of a hole extracting agent forces holes to remain in SiNWs. ${ }^{29}$ Ongoing injection of electrons increases the number of holes within SiNWs leading to a transient electrical



Fig. 6 The sensing mechanism of $\mathrm{p}$-SiNWs/ITO before and after importing $\mathrm{NO}_{2}$ under visible light illumination, respectively.

polarization corresponding to (dynamic) accumulations of holes (SiNWs) and electrons (ITO). This is a self-limiting process that continues until the formed electric field blocks any further injections of electrons, leading to generation of a photocurrent in the external circuit.

Under $\mathrm{NO}_{2}$ atmosphere, the electrons accumulated at the SiNWs/ITO interface can be trapped by $\mathrm{NO}_{2}$ molecules, the trapped electrons at the junction are subsequently compensated by bulk ITO electrons, causing a decrease in the apparent donor density within the metal oxide ( $N_{\mathrm{D}}$ decreases). The equations of the semiconductor quantum theory can be used to support the above-described mechanism. ${ }^{29,30}$ The relation between the donor concentration $\left(N_{\mathrm{D}}\right)$ and the built-in potential $V_{\mathrm{bi}}$ in a p-n junction is expressed by

$$
V_{\mathrm{bi}}=\frac{K T}{Q} \ln \left(\frac{N_{\mathrm{D}}^{\mathrm{ITO}} N_{\mathrm{A}}^{\mathrm{Si}}}{N_{\mathrm{i}}^{\mathrm{ITO}} N_{\mathrm{i}}^{\mathrm{Si}}}\right)
$$

where $N_{\mathrm{D}}, N_{\mathrm{A}}$ and $N_{\mathrm{i}}$ are donor, acceptor and intrinsic doping level, which corresponds with the free carrier concentration in the impurity ionization regime, $K, T$ and $Q$ are Boltzmann constant, absolute temperature and electron charge, respectively. The decrease in the concentration of electrons in ITO in presence of $\mathrm{NO}_{2}$ can be regarded as a decrease in the effective concentration of donor impurities $N_{\mathrm{D}}$ in the n-type ITO. According to eqn (1), the reduction of $N_{\mathrm{D}}$ leads to a decrease of the $V_{\mathrm{bi}}$ value in $\mathrm{NO}_{2}$ atmosphere. The open circuit voltage $\left(V_{\mathrm{oc}}\right)$ that appears across the terminals of a $\mathrm{p}-\mathrm{n}$ junction under illumination is caused by the ability of this built-in potential to separate the electron and hole pairs generated within and close to the depleted region. Thus, there exists a positive correlation between the photovoltage values (and in particular the opencircuit voltage $V_{\mathrm{oc}}$ ) and $V_{\mathrm{bi}},{ }^{31,32}$ so the open-circuit $I$ which determined by $V_{\mathrm{oc}}$ is also proportional to $V_{\mathrm{bi}}$. This can elaborate why the current decrease in presence of $\mathrm{NO}_{2}$.

\section{Conclusion}

In summary, we have fabricated and investigated a photodriving self-powered $\mathrm{p}$-SiNW/ITO heterojunction device, in which the tip of the SiNWs was directly in contact with the ITO electrode and gas analytes could diffuse into the SiNW array. Under visible light illumination, the device showed high 
photosensitivity and ultrafast response speeds under zero bias voltage, and displayed a distinctive binary response under a small forward bias. The device can sensitively and quantitatively respond to $\mathrm{NO}_{2}$ gas without the need of external power. In comparison with chemiresistive sensors based on SiNWs only, the sensitivity of the self-powered sensor was improved. More importantly, the photodiode-structure nanosensor can persistently output electrical power by harvesting light energy, and in the meantime monitor the surrounding gas without a power supply. This work may have a huge application in the fields of environmental sensing, biosensing, and optoelectronic integrated circuits and bring about a novel idea and a facile method to develop self-powered gas microsensors based on the rational design of $\mathrm{p}-\mathrm{n}$ heterojunctions using nanomaterials.

\section{Experimental}

\section{Materials}

P-type silicon wafers (1 00 crystal, boron-doped, 5-10 $\Omega \mathrm{cm}$ ) and N-type Si $\left(\begin{array}{lll}1 & 0 & 0\end{array}\right)$ wafers (phosphor-doped, 1-10 $\Omega \mathrm{cm}$ ) were purchased from Beijing XiHe Ruida Technology Co. (China). ITO $\left(<15 \mathrm{ohm} \mathrm{sq}^{-1}\right)$ was purchased from Zhuhai Kaivo Optoelectronic Technology Co. (China). $\mathrm{HF}(40 \%), \mathrm{H}_{2} \mathrm{O}_{2}(30 \%)$, $\mathrm{AgNO}_{3}(>99.9 \%), \mathrm{HNO}_{3}(65-68 \%)$, ethanol ( $\left.\geq 99.7 \%\right)$, acetic acid $(\geq 99.5 \%)$ were purchased from Sinopharm Chemical Reagent Co. (China). The standard $\mathrm{NO}_{2}$ gas sample with concentration of $16.8 \mathrm{ppm}$ (diluted in $\mathrm{N}_{2}$ ) was purchased from Hangzhou Xinshiji Mixed Gas Co. (China).

\section{Preparation of SiNWs array}

Si wafers were cut into pieces with dimension of $5 \times 5 \mathrm{~mm}^{2}$. The silicon nanowires (SiNWs) were prepared by metal assisted chemical etching (MACE) similar to the method as described previously. ${ }^{33}$ Briefly, the Si wafers were immersed in $0.008 \mathrm{M}$ $\mathrm{AgNO}_{3}$ and $4.8 \mathrm{M} \mathrm{HF}$ for $2 \mathrm{~min}$ at room temperature. After the $\mathrm{Ag}$ nanoparticles formed, the wafers were washed with deionized water to remove the remained $\mathrm{Ag}^{+}$ions, and then etched in 4.8 $\mathrm{M} \mathrm{HF}$ and $0.4 \mathrm{M} \mathrm{H}_{2} \mathrm{O}_{2}$ for $2 \mathrm{~h}$ under room temperature. Then the wafers were washed repeatedly with deionized water and immersed in the $\mathrm{HNO}_{3}$ water solution $(1: 1 \mathrm{v} / \mathrm{v})$ for $30 \mathrm{~min}$ to dissolve the remained $\mathrm{Ag}$ particles. After that the as-prepared SiNWs were immersed in $3.3 \% \mathrm{HF}$ solution for $10 \mathrm{~min}$ to remove the oxide layer. Finally, it was cleaned with deionized water and ethanol and dried under $\mathrm{N}_{2}$ flow.

\section{Characterization and gas detection system}

The morphologies of the synthesized SiNWs arrays were investigated using field-emission scanning electron microscopy (FESEM, Hitachi SU 8010) with an operating voltage of $5 \mathrm{kV}$ and magnification of 2500 were applied.

The SiNWs/ITO structure is basically constructed by compressing the transparent ITO and the tip of SiNWs with proper pressure. In order to investigate the performance of gas sensing, the SiNWs/ITO sensor was sealed into a chamber with a window for light illumination and the volume of the chamber was $\sim 2$ $\mathrm{cm}^{3}$. The concentration of $\mathrm{NO}_{2}$ gas sample was adjusted by mixing the standard $\mathrm{NO}_{2}$ sample (16.8 ppm) with $\mathrm{N}_{2}$ at a specific ratio controlled by an automatic gas mixing apparatus (National Institute of Metrology, China). The total flow rate of the mixture

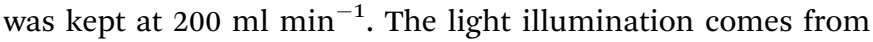
a LED (S2000, Nanjing Hecho Technology Co., China) emitting white light. The light intensity in the chamber was $20 \mathrm{~mW} \mathrm{~cm}^{-2}$, which was measured by an optical power meter (SGN-1, Shanghai Shuoguang Optoelectronic Technology Co., China). The sealed chamber was kept at room temperature. The current-voltage $(I-V)$ data of SiNW/ITO heterojunction were recorded by a Keithley 6487 digital source-meter.

\section{Conflicts of interest}

There are no conflicts to declare.

\section{Acknowledgements}

This work was supported by National Science Foundation of China (No. 21575127), and Zhejiang Provincial Natural Science Foundation of China (No. Z15B050001).

\section{Notes and references}

1 J. A. Rogers and Y. Huang, Proc. Natl. Acad. Sci. U. S. A., 2009, 106, 10875-10876.

2 L. Yuan, J. Dai, X. Fan, T. Song, Y. T. Tao, K. Wang, Z. Xu, J. Zhang, X. Bai and P. Lu, ACS Nano, 2011, 5, 4007.

3 L. Yuan, Y. Tao, J. Chen, J. Dai, T. Song, M. Ruan, Z. Ma, G. Li, L. Kang and X. Zhang, Adv. Funct. Mater., 2011, 21, 21502154.

4 Z. L. Wang, Adv. Mater., 2012, 24, 280-285.

5 Z. L. Wang, Adv. Funct. Mater., 2008, 18, 3553-3567.

6 X. Xu, T. Ding, L. Yuan, Y. Shen, Q. Zhong, X. Zhang, Y. Cao, B. Hu, Z. Teng and G. Li, Adv. Energy Mater., 2012, 2, 13281332.

7 Z. L. Wang, Nano Today, 2010, 5, 512-514.

8 X. Xiao, L. Yuan, J. Zhong, T. Ding, Y. Liu, Z. Cai, Y. Rong, H. Han, J. Zhou and Z. L. Wang, Adv. Mater., 2011, 23, 5440-5444.

9 Z. L. Wang, Sci. Am., 2008, 298, 82-87.

10 X. Xue, Y. Nie, B. He, L. Xing, Y. Zhang and Z. L. Wang, Nanotechnology, 2013, 24, 225501.

11 R. Zhang, L. Lin, Q. Jing, W. Wu, Y. Zhang, Z. Jiao, L. Yan, R. P. Han and Z. L. Wang, Energy Environ. Sci., 2012, 5, 8528-8533.

12 Y. Hu, C. Xu, Y. Zhang, L. Lin, R. L. Snyder and Z. L. Wang, Adv. Mater., 2011, 23, 4068-4071.

13 N. Cui, W. Wu, Y. Zhao, S. Bai, L. Meng, Y. Qin and Z. L. Wang, Nano Lett., 2012, 12, 3701-3705.

14 A. Gad, M. W. Hoffmann, O. Casals, L. Mayrhofer, C. Fàbrega, L. Caccamo, F. Hernández-Ramírez, M. S. Mohajerani, M. Moseler and H. Shen, ACS Sens., 2016, 1, 1256-1264.

15 N. M. Vuong, N. M. Hieu, H. N. Hieu, H. Yi, D. Kim, Y.-S. Han and M. Kim, Sens. Actuators, B, 2014, 192, 327-333. 
16 K. Peng, X. Wang and S.-T. Lee, Appl. Phys. Lett., 2008, 92, 163103.

17 Y. Cao, J.-H. He, J.-L. Zhu and J.-L. Sun, Chem. Phys. Lett., 2011, 501, 461-465.

18 Y. Cui, X. Duan, J. Hu and C. M. Lieber, J. Phys. Chem. B, 2000, 104, 5213-5216.

19 M. Zhu, X. Li, S. Chung, L. Zhao, X. Li, X. Zang, K. Wang, J. Wei, M. Zhong and K. Zhou, Carbon, 2015, 84, 138-145.

20 G. Li, L. Liu, G. Wu, W. Chen, S. Qin, Y. Wang and T. Zhang, Small, 2016, 12, 5019-5026.

21 M. W. Hoffmann, L. Mayrhofer, O. Casals, L. Caccamo, F. Hernandez-Ramirez, G. Lilienkamp, W. Daum, M. Moseler, A. Waag and H. Shen, Adv. Mater., 2014, 26, 8017-8022.

22 M. W. Hoffmann, A. E. Gad, J. D. Prades, F. HernandezRamirez, R. Fiz, H. Shen and S. Mathur, Nano Energy, 2013, 2, 514-522.

23 Y. Jia, Z. Zhang, L. Xiao and R. Lv, Nanoscale Res. Lett., 2016, 11, 299.
24 H. Fang, X. Li, S. Song, Y. Xu and J. Zhu, Nanotechnology, 2008, 19, 255703.

25 G. Yuan, H. Zhao, X. Liu, Z. S. Hasanali, Y. Zou, A. Levine and D. Wang, Angew. Chem., Int. Ed., 2009, 48, 9680-9684.

26 M. Shao, L. Cheng, X. Zhang, D. D. D. Ma and S.-t. Lee, J. Am. Chem. Soc., 2009, 131, 17738-17739.

27 Y. Cui, Z. Zhong, D. Wang, W. U. Wang and C. M. Lieber, Nano Lett., 2003, 3, 149-152.

28 H. Kim, a. C. Gilmore, A. Pique, J. Horwitz, H. Mattoussi, H. Murata, Z. Kafafi and D. Chrisey, J. Appl. Phys., 1999, 86, 6451-6461.

29 J. Piris, A. J. Ferguson, J. L. Blackburn, A. G. Norman, G. Rumbles, D. C. Selmarten and N. Kopidakis, J. Phys. Chem. C, 2008, 112, 7742-7749.

30 S. Sze, Physics of semiconductor devices, 2007, vol. 3.

31 B. Kaplan, Phys. B, 2004, 351, 90-95.

32 X. Chao, Y. Ruo-He and G. Kui-Wei, Chin. Phys. B, 2011, 20, 057302.

33 D. Liu, L. Lin, Q. Chen, H. Zhou and J. Wu, ACS Sens., 2017, 2, 1491-1497. 